\title{
Operando and in situ in a TEM imaging in a cryogenic temperature range
}

Martial Duchamp ${ }^{1}$, Joseph Vas ${ }^{2}$, Reinis Ignatans ${ }^{3}$, Aaron David Mueller ${ }^{2}$, Rohit Medwal ${ }^{2}$, Rajdeep Rawat $^{2}$ and Vasiliki Tileli ${ }^{4}$

${ }^{1}$ Nanyang Technological University (NTU), United States, ${ }^{2}$ Nanyang Technological University (NTU), Singapore, Singapore, ${ }^{3}$ Institute of Materials, École Polytechnique Fédérale de Lausanne, United States, ${ }^{4}$ Institute of Materials, École polytechnique fédérale de Lausanne, Lausanne, Switzerland

We present two temperature dependent operando studies inside the TEM, namely, the evolution of ferroelectric and ferromagnetic domains walls, in $\mathrm{BaTiO}_{3}(\mathrm{BTO})$ and $\mathrm{Y}_{3} \mathrm{Fe}_{5} \mathrm{O}_{12}$ (YIG) respectively. Continuous imaging of the sample as a function of electric or magnetic fields at different temperatures allows us to quantify the activation energy of the specific pinning sites. Hence, we study the pinning of the domains with different stimuli like temperature, externally applied magnetic field, electric fields and currents to better manipulate the domain walls. These studies in a continuous cryogenic temperature range will allow us to unravel the fundamental understanding of these physical phenomena at nanometer scale.

The TEM lamellae, used for these studies, were transferred from bulk YIG and BTO single crystal sample, onto the MEMS chip and electrically connected [1, 2]. The high-resolution cryogenic imaging using MEMSbased holder have been shown to delay the irradiation damage [3], but the combination of low temperature imaging in a continuous variable range under operando conditions is still be be demonstrated. The study of ferroelectric domain wall pinning in BTO crystal, a prototypical ferroelectric system, from its low temperature orthorhombic phase continuously up to its centrosymmetric high temperature phase is shown to be possible inside a TEM under externally applied electrical field $[4,5]$. Additionally, the micrographs show that upon heating the $180^{\circ}$ domain walls of the tetragonal phase are pinned at the same sites as their low temperature orthorhombic counterpart while this effect is not observed upon cooling, i.e., the domains of the tetragonal phase have merged upon cooling but do not split upon annealing across the phase transition to the orthorhombic structure. The operando TEM experiments allows us to study the domains formation across phase transitions under externally applied electrical field as a function of cryogenic temperature. 
The propagation of magnetic domain walls in YIG, a magnetic insulator, is also studied by varying the temperature (from $\sim 120 \mathrm{~K}$ to $\sim 400 \mathrm{~K}$ ), external magnetic field and electric current [6]. We use Lorentz TEM conditions (magnetic field-free conditions at the sample location) to image the displacement of domain walls in YIG crystal, first under different applied magnetic fields and then by applying different current densities in the adjacent metallic layer. We establish a three dimensional magnetic field, current density and temperature phase diagram of the control of magnetic domains based on direct observations using Lorentz TEM imaging. These outputs can then be used to improve the design of spintronics based switching devices which play a critical role in designing low loss memory devices for future computational era.

\section{References}

[1] Duchamp, M.; Xu, Q.; Dunin-Borkowski, R. E. Convenient Preparation of High-Quality Specimens for Annealing Experiments in the Transmission Electron Microscope. Microscopy and Microanalysis. 2014, 20 (06), 1638-1645.

[2] Jeangros, Q.; Duchamp, M.; Werner, J.; Kruth, M.; Dunin-Borkowski, R. E.; Niesen, B.; Ballif, C.; HesslerWyser. A. In Situ TEM Analysis of Organic-Inorganic Metal-Halide Perovskite Solar Cells under Electrical Bias. Nano Letters. 2016, 16 (11), 7013-7018.

[3] Tyukalova, E.; Duchamp, M. Atomic Resolution Enabled STEM Imaging of Nanocrystals at Cryogenic Temperature. J. Phys. Mater. 2020, 3 (3), 034006.

[4] Ignatans, R.; Damjanovic, D.; Tileli, V. Local Hard and Soft Pinning of $180^{\circ}$ Domain Walls in $\mathrm{BaTiO}_{3}$ Probed by in Situ Transmission Electron Microscopy. Phys. Rev. Materials. 2020, 4 (10), 104403.

[5] Ignatans, R.; Damjanovic, D.; Tileli, V. Local Hard and Soft Pinning of $180^{\circ}$ Domain Walls in $\mathrm{BaTiO}_{3}$ Probed by in Situ Transmission Electron Microscopy. Phys. Rev. Materials. 2020, 4 (10), 104403.

[6] Medwal, R.; Chaudhuri, U.; Vas, J. V.; Deka, A.; Gupta, S.; Duchamp, M.; Asada, H.; Fukuma, Y.; Mahendiran, R.; Rawat, R. S. Magnetoimpedance of Epitaxial $\mathrm{Y}_{3} \mathrm{Fe}_{5} \mathrm{O}_{12}$ (001) Thin Film in Low-Frequency Regime. ACS Appl. Mater. Interfaces. 2020, 12 (37), 41802-41809. 